\title{
ZUR TEXTKRITIK DES REINHART FUCHS.
}

Die dichtung des Elsässers Heinrich des Glichezare ist uns in ihrer ursprunglichen gestalt nur bruchstlickartig in einer handschrift aus dem ende des 12. jahrhunderts erbalten. (J. Grimu sendschreiben an Karl Lachmann uber Reinhart Fuchs, Leipzig 1840.) Vollständig dagegen kennen wir das gedicht in einer bearbeitung des 13. jahrhunderts, welche uns in zwei handschriften uberliefert ist, in einer Heidelberger (cod. palat. 341) und in einer Kalocsaer. Aus der letzteren ist Reinhart Fuchs von Mailath und Köffinger in dem 'Kolocsaer codex altdeutscher gedichte, Pesth 1817', doch fehlerhaft abgedruckt. Die Heidelberger handschrift haben wir bezuglich der erwähnten dichtung zunăchst aus Grimms edition kennen gelernt. Beide codices 1 ), pergamenthandschriften aus dem 14. jahrhunderte, babe ich collationiert und das resultat dieser collation in meiner ausgabe des Reinhart - Fuchs (Pauls altdeutsche textbibliothek) verwertet. Die handschriften sind nicht bloss nach ihrer äussern einrichtung und ihrem schriftductus, sondern auch nach ihren sprachlichen und graphischen eigentlumlichkeiten im ganzen ubereinstimmend und weisen nach Mitteldeutschland, vielleicht nach Ostfranken.

Es ist einigemale hehauptet worden, der Kalocsaer codex (K.) sei von dem Heidelberger (P.) abgeschrieben. Auch Schönbach ist in seinem jüngst veröffentlichten wertvollen beitrage zur Reinhartkritik (Zf. f. d. a. XXIX, s. 47 ff.) der ansicht, K. sei 'nur eine copie von $P$ und zwar mechanisch angefertigt und ohne dass eine andere handschrift zu rate gezogen

1) Ueber beide codices $\nabla$ gl. man ubrigens Fundgraben I, s. 240, v. d. Hagen, Gesammtabenteuer III, s. 752; Haupt, Lieder und blichlein s. IX $\mathbf{f}$. 
wäre.' Ich habe auf Schönbachs darlegung hin mir die sache nochmals angesehen, muss aber bekennen, dass ich seine anschauung nicht acceptieren kann. Meine ansicht geht vielmehr dahin, dass P. und K., wie im ganzen, so bezuglich Reinharts unabhängig von einander sind, doch auf eine und dieselbe quelle zurückweisen. Ich will davon absehen, dass die zahl und anordnung der stiucke in beiden handschriften nicht mit einander tubereinstimmt, schon der text spricht gegen eine abschrift des cod. K. von P. Ganz richtig hat sich M. Haupt in der vorrede zu seiner ausgabe der 'Lieder und bthchlein und des armen Heinrich' s. IX f. uber die erwähnte behauptung geäussert: diese 'wird weder im armen Heinrich, noch in den andern gedichten, von denen ich den text beider kenne, bestätigt: beide können abschriften einer verlorenen sein'. Dem stimme ich auch vom standpunkte unseres gedichtes bei. Mögen auch die differenzen zwischen $P$. und $K$. im ganzen unwesentlich sein, alles in K. lässt sich doch nicht aus $\mathrm{P}$. erklären. So bietet K. z. b. 326 ff. nur den ausdruck valle, während $P$. widerholt dafur druhe, druch einsetzt. Das ursprungliche kann nur valle sein, wie $331 \mathrm{f}$. der reim valle : Galle beweist. Diese sachlage deutet aber entschieden nicht auf die abhängigkeit des cod. K. von $P$. bin. 1065 uberliefert $K$. usgezeuge (: urleuge), das einen reinen reim gibt, wogegen $\mathrm{P}$. ungezewe (: urleuge), also einen ungenauen reim bietet. Dagegen will ich nicht lăugnen, dass cod. $P$., mag er auch sonst, gleich $K$, entstellungen aufweisen, den bessern text uberliefert und, wie eine vergleichung mit dem alten Reinhart lehrt, dem ursprtinglichen texte der bearbeitung etwas năher steht, als K. Auch der name des dichters ist in P. treuer gegeben, als in $\mathrm{K}$. Vgl. $1786 \mathrm{u} .2250$.

Was die sprache der bearbeitung anlangt, so ist es nur sehr weniges, was sich hieruber mit sicherheit feststellen lassst. Deshalb hat es auch seine schwierigkeit, den text ganz in dem wortlaute, herzustellen, den ihm der bearbeiter gegeben hat. Und den handschriften konnte man in dieser hinsicht doch nicht unbedingt folgen.

Auch die metrischen verhăltnisse liegen im Reinhart Fuchs im argen. Doch hat auch nach dieser richtung Schönbach in der angezogenen publication die textkritik unserer 
dichtung, namentlich durch den nachweis zweisilbiger senkungen, erheblich gefördert.

Bei der gestaltung des textes in meiner ausgabe habe ich mich, nicht bloss im hinblicke auf die zwecke der 'altdeutschen textbibliothek', sondern hierin auch Schönbachs auseinandersetzungen folgend, enger an die handschriftliche uberlieferung angeschlossen. Ich habe $P$. den vorzug gegeben, doch nicht unbedingt. An einigen stellen, wo K. das bessere zu haben schien, habe ich vielmehr diesen codex berlucksichtigt. Ebensowenig meinte ich den text des alten gedichtes (S) von der textgestaltung der bearbeitung ferne halten $\mathrm{zu}$ sollen, zumal dort wo die bearbeitung corrumpierte stellen bietet, im alten gedichte aber alles plan ist. Wo bestimmte grlinde $\mathrm{zu}$ einer anderung nicht vorlagen, habe ich die sprachliche gestalt des Grimm'schen textes beibehalten.

Soviel im allgemeinen. Ueber alles weitere mögen die folgenden bemerkungen orientieren.

20 zu Ruotzela vgl. Schönbach a. a. o. - 34 klammer mit Schönbach. - 36 babe scheint nur von den schreibern eingefuhrt. Es steht bereits oben v. 28 und ist hier wol uberflussig. - 40 Mit liet (P.) und het (K.) ist nicht viel anzufangen. Auch Grimms riet (Reinhart an den lîp) will mir nicht behagen. Ich setze, da ich vorläufig nichts besseres weiss, den gie Reinhart an den lîp. - 46 brahtern für brahten der cod. 49 sinen der cod. ist doch wol nur für einen verschrieben. Ich setze daher dieses. Fur senete der cod. und bei Grimm nehme ich mit Schönbach smucte auf, obwol ich einem denete sich nicht ganz abgeneigt wăre. Im Rom. de Renart heisst es (Martin II, 1. br. v. 60): moult se defripe. - $53 \mathrm{f}$. interpunction mit Schönbach. - 54 könnte diu henne vom bearbeiter herruhren und gar wol stehen, da Pinte hier zum ersten male vorkommt. Der vers würde dann einen dreisilbigen auftact, also nichts ungewöhnliches haben. Doch habe ich diu henne nicht in den text gesetzt, dafür aber auch nicht Schönbachs ver (Pinte) aufzunehmen mich entschliessen können. - 63 weiss ich uf erwarten nicht zu bessern. - 69 muss mit Schōnbach einem geschrieben werden. - 135 schrei (PK.) ist nicht gut auszulassen, da die drei folgenden zeilen sich erst aus diesem worte recht erklären. 136 nehme ich von Grimm wan auf, zugleich aber aus den 
cod. auch danne. - 143 lât für lazet der cod. mit Grimm ef. 89. Die bearbeitung zeigt tiberhaupt eine besondere vorliebe fur contrahierte verbalformen. Von lâzen fuhre ich an: lân: getân 11 f. 1205 f. 2059 f., : hân 291 f. 601 f., : stan 1985 f., : gân 739 f. $821 \mathrm{f}$, lât : rât 2237 f. $-147 \mathrm{f}$. die stelle macht schwierigkeiten. Aber ich möchte nicht, wie Grimm tut, den reim dô : vrô ganz beseitigen, denn er ist in der bearbeitung beliebt. Vgl. $49 \mathrm{f}$. 243 f. 321 f. 609 f. 679 f. 1019 f. 1225 f. 1489 f. 1689 f. 1795 f. $2065 \mathrm{f} .2103 \mathrm{f}$, d do : unvrô $405 \mathrm{f}$. $1453 \mathrm{f} .2227 \mathrm{f}$. Hier freilich erwartet man $d \hat{a}$. Aber wol nicht ohne grund vermutet Paul: 'ungerne dâ könnte nach dem dialecte auf vrô reimen'. Den bearbeiter denke ich mir im Elsass oder im westlichen Mitteldeutschland. Gibt man dô : vrô auf, so kehrt man wol am besten zu Grimms $d \hat{a}: s \hat{a}$ zurbick. Als er im entweich der cod. ist schwer aufrecht zu erhalten. Ich schreibe mit Schönbach: als er im entleip. Die entsprechende stelle im Rom. de Renart (Martin II br., v. 435) lautet: Quant cil sentilache la boce. wart ist fur want verschrieben. sam steht für sân, sâ. Dies ist, wie Schönbach mit recht hervorhebt, ursprunglich und deshalb ist es auch nicht zu tilgen. Dass der vers holperig und anormal ist, darf bei einem machwerke des wenig befăhigten bearbeiters nicht befremden. - 157 das fragezeichen bei Grimm ist uberflussig. - 200 rührt sin gavatere offenbar nur von einem schreiber her und Grimm tat recht, es zu eliminieren. Das wort kehrt ohnehin 202 wider. - 203 Ich setze ein mist mit P., ubrigens hat auch K. 209 daz mist. -209 f. fur begripfte : entwischte ist mit Grimm (anmerk.) begripfte : entslipfte anzunehmen. - $249 \mathrm{f}$. ich schreibe hier und in ăhnlichen reimen niet und nehme es nicht blos, wie Schönbach zu $751 \mathrm{f}$. es tut, fur den bearbeiter, sondern auch fur den dichter an. $1759 \mathrm{f}$. uberliefert P. geradezu: diet : niet. Ausserdem fuhre ich die reime an: liet : niht 511 f. 533 f., geriet : niht 751 f. 769 f. 1327 f. 2119 f., diet : niht $1297 \mathrm{f} .1809 \mathrm{f}$. In diesen fällen setze ich tberall niet und dem entsprechend 455 in dem reime iht : diet ein iet. Die meisten der angefuhrten reime sind von dem bearbeiter aus der alten dichtung übernommen. Denn $1671 \mathrm{f}$. und $1759 \mathrm{f}$. ist sogar in S. diet : niet luberliefert. und ein niet, iet ist nicht im widerspruch mit der sprache des dichters. Weinhold, Al. gr. §. 63. Indem der bearbeiter diese reime gelten liess, tat or Beiträge zur geschichte der deutschen sprache. XT. 
jedoch seiner sprache keinen zwang an, denn er selbst bildet an stellen, wo er von dem alten gedichte abweicht, so bestimmt $769 \mathrm{f}$., solche reime und sie stehen mit seiner heimat gewiss in einklange. Weinhold, Mhd. gr. ${ }^{2}$ \& 494. Germ. XXIX, s. 262. - 258 und 264 lese ich mit Schönbach tratneve. 282 f. schreibe ich statt vil der vedern, vier der vedern nach Rom. de Renart (Martin II br., v. 991) qatre des penes. - 284 gegen Grimm mit PK., vgl. Schönbach. - 285 uberliefern PK. vihen. Das ist aber nur ein schreibfehler, da eine solche sinnlosigkeit selbst dem bearbeiter nicht zuzumuten ist. Ich nehme Grimms conjectur imbîzen auf. - 300 setze ich iu für P. euch K. uch und so auch spăter im gegensatze zu Schönbach, in ubereinstimmung mit Paul. Denn die handschriften sind in dieser vertretung des dat. durch den acc. nicht consequent und so kann man doch nicht gewiss entscheiden, ob dieselbe blos den handschriften, oder auch dem bearbeiter, ja selbst dem dichter zukommt. 329 interpunction mit Schönbach. - 345 Von der zutat in K. ist naturlich abzusehen. - 357 f. P. bietet Lucifere : schiere einen reim, der mitteldentsch rein sein könnte. Vgl. Weinhold, Mhd. gr. ${ }^{2} \S 135$. K. hat statt dessen Lucifere : gevere, was noch bestimmter mitteldeutsch ist. - 386 lasse ich den wolf der cod. aus, wie 54 die henne. - 415 in daz lant, wio Schōnbach mit PK. unter hinweis auf 574 schreibt. - 447 nôt mit PK. Vgl. hierzu die reime: nôt : tôt 273. : rôt 283. u. 8. w. - 479 f. mit K. und Grimm kleine : beine. beine, md. pluralform. Weinhold, Mhd. gr. ${ }^{2} \& 454$. -489 ist es nicht nōtig, mit Schönbach aus $\mathrm{P}$. spilinde aufzunehmen. Das zweite $i$ gehört doch wol nur dem mitteldeutschen schreiber an. - 500 wellet statt wollet der cod. mit Weinhold, Mhd. gr. ${ }^{2}$ §421. - 511 abermals eine wertlose einschaltung in $\mathrm{K}$. 521 nehme ich mit Grimm die rtickumgelautete form schancte. Ruckumlaut ist in der bearbeitung mehrfach durch den reim belegt: (geluste :) kuste 180, gerant (: want) 59, (: zehant) 793, gesant (: zehant) 1322. 1889, erkant (: elefant) 1334, genant (: lant) 1241. — 559 mit P. leiste. leist, stmf., sonst noch bei Reinfried von Braunschweig. - 562 Grimm: genuogez brôt, ich wähle genuoc brôt. Cf. 1779 f. brôt : nôt. Oder sollte man schreiben: genuoc unz in den tôt? tôt : nôt hăufiger reim im gedichte. Cf. auch Nib. 638, 3. - 594 mit den cod. weste, obwol einmal wiste 
(: liste) $506 \mathrm{im}$ reime steht. wiste, weste finden sich ja häufig lei éinem dichter nebeneinander. - 626 P. weiz got lasse ich fallen, in $K$. fehlt es auch. Zudem erscheint es 629 wider und zwar passender. Hier gehört es blos dem schreiber von'P. an. Fü ich travete (trowet) ime an treven (trimen) wol mit Schönbach ich drouwete ime entriuwen wol. - $631 \mathrm{zu}$ lat vgl. oben 143. 647 Ich sehe nicht ein, weshalb der bearbeiter das bessere, bezeichnendere ersmacte, das noch in $\mathrm{S}$. steht, gegen smacte eingetauscht haben sollte und nehme daher jenes auf. - 648 ahd nach PK. - 653 dar în bôzen mit PKS. - 649 f. der bearbeiter hat sich uber den unreinen reim des ursprunglichen gediclites spîse : wîsen nicht hinweghelfen können. Ein schwf.spîse gibt es, eoviel ich sehe, nicht. Andero reimo mit uberschlissigem $-n$ sind: laden : schade $1449 \mathrm{f}$, ritze : sitzen $931 \mathrm{f}$, burne : misselungen $955 \mathrm{f}$., alumbe : tumben $1757 \mathrm{f}$. (? S. rein: alumbe : tumbe). - 654 der aus S. zu ergänzen. v.655 PK. sprach (ohne er, das Grimm hat) deutet darauf hin. - 655 und 659 zu gân und varn in S. und bern in PK. of. Schönbach. - 657 wol hat in S. die richtige stelle, die ich deshalb auch acceptiere. - 658 ist mit Schönbach den sin dîn (PK.) zu belassen. - 673 nehme ich aus $P$. da maht lihte vergeben auf. Von der einschiebung in $\mathrm{K}$. ist hier abzusehen. - 674 das handschriftliche vürbaz macht den vers $\mathrm{zu}$ einem funfhebigen. Das ist aber kein grund das passende wort aufzugeben. $T 675$ getete der cod. kann auch fur getaete genommen werden, da $e$ in den cod. auch fur ae steht. - 680 mit Schönbach ginen gegen Grimms ginen. - 6£4 hinne mit Wackernagel. - 699 paradis, wie 898 und 912 PKS. - 703 PK. und S. haben also, das ich auch bolialte. - 713 dinne mit Grimm wie PK. 765 und 869. - 714 ich bleibe bei Grimms sit, da ich nicht weiss, ob sint wirklich dem bearbeiter oder blos den cod. zukommt. - 725 besser scheint mir der ausdruck in S.: die bruodir leitense drîn. Doch ich bleibe bei der uberlieferung in $\mathrm{PK}$. -730 passt loch in $\mathrm{S}$. metrisch besser, als gruobe, das PK. uberliefern. Aber es ist wahrscheinlich, dass, wie Schönbach - im manuscript des erwăhnten aufsatzes - vermutet, nicht loch der urspringliche ausdruck sei, sondern ein bezeichnenderer hier gestanden habe. In dem entsprechenden siebenb. tiermärchen wird de lâm uberliefert. (Wolff-Haltrich, Zur volkskunde der Siebenburger Sachsen, Wien 1884, s. 38). - 
734 hat der bearbeiter den vers, um seine form zu bessern, dem sinne nach verschlechtert. Der alte vers ist klarer und der folgende schliesst. sich besser an den alten an. - 739 hie $\hat{\imath} n$ mit PS. - 740 statt stürmen bei Grimm (nach PK.) ist mit Schönbach stüren zu setzen. - 745 fehlt er sprach (PK.) in S. Auch in der bearbeitung bleibt es mitunter aus. Uebereinstimmend in SPK.: 739. 747. 748. 909. 929. 1539 u. a. o. Ich lasse es an unserer stelle weg und so durfte es auch an andern stellen zu eliminieren sein (man vgl. W. Grimm, Graf Rudolf s. 13). Auch E. Schröder sieht im Anegenge s. 19 ein solches er sprach als heraustretend aus dem verse an und Amelung erklärt es Zs. f. d. philol. III, 8. 268 in den von ihm betrachteten gedichten auch fur uberflussig. - 750 drinne mit PKS. - 763 fehlt daz (PK.), welches auch Grimm weglässt, in S. Nichtsdestoweniger setze ich es. - 769 Schönbachs conjeçtur kachen für Grimms zocken ist fein, aber zu fein, wie mir scheint, für den bearbeiter. Ich wage deshalb nicht, sie in den text zu setzen, um so mehr als zocken, wofur wol die cod. PK. kochen verschrieben haben, immerhin noch einen erträglichen sinn bietet. - 784 P. ungelat, K. ungelabt, J. Grimm im text des RF. ungelabet, anmerk. ebenda: ungejaget. Ich wählte dies, das in PK. verschrieben sein kann und an S. eine stutze findet (an jagin kertir sinen sin). - 786 var mit PK. und Schönbach gegen vart in S. - 795 erbeizte mit PKS. - $800 \mathrm{f}$. offenbar vom bearbeiter eingeschoben, um den unreinen reim zu beseitigen. $-807 \hat{a f}$ den rucke mit $\mathrm{PK}$. und Schönbach. -808 vgl. hierzu Grimm RF. anmerk. und Schönbach. - 813 glete im aber mit PKS., obwol der vers schlechter wird als bei Grimm, ef. Schönbach. - $821 d a$, wie S. $d o$ in PK. bietet nur eine mudartliche verdumpfung des $a$ und besagt dasselbe. $-833 \mathrm{ff}$. ich setze consequent burne. In den handschriften steht - mit ausnahme von 851 - ubereinstimmend bald brunne, bald burne. Die form burne ist sowol dem elsåssischen, als dem mitteldeutschẻn eigen. Vgl. Weinhold, Mhd. gr. ${ }^{2} \S 214$, Al. gr. § 197, Grimm, Deutsches wtb. II, s. 433, Schönbach a.a. o. - 837 schreibe ich, um der handschriftlichen tuberlieferung in PK. näher zu bleiben, mit Schönbach sich verginte, doch wäre ich auch nicht abgeneigt, aus S. ergouchete aufzunehmen, das jedenfalls besser in den sinn passt. - 847 ist vor kleinen ein niht zu ergänzen. S. hat michelin danch. -855 fuge ich mir aus S. ein. -862 
wol nach PKS. (Grimm wole). - $865 \mathrm{f}$. tief : gief. Dieser reim ruhrt vom bearbeiter her, da $\mathrm{S}$. hier einen andern text hat. Der reim ist aber eben deshalb von bedeutung fur die herkunft des bearbeiters. Denn gief ist bisher blos aus mitteldeutschen quellen belegt, aus dem Renner, dem Passional, aus Rüdiger von Münner (Gesammtabenteuer 3, 62, 720), den Kolmarer meisterliedern, aus Heinrich und Kunigunde, Jeroschin, dem Väterbuch (Zingerle Findlinge 2,127) und aus Aristotelis heimlichkeit (Lexer III, s. V). Auch giefen findet sich blos in einem mitteldeutschen denkmal, dem jungern Titurel. - 885 Für PK. ergetzet setzt S. irgouchet. - 908 mit dem cod. verbrant sô und nicht mit Grimm verbrennet sô. Das part. praet. bat nach den reimen (vgl. oben zu v. 521) auch sonst ruckumlaut und das fehlen der letzten senkung ist ja in der bearbeitung, wie in S., auch nicht auffallend. - 904 mit Grimm Send anm. und Schönbach roup, obwol sich hier auch ruowe nemen, das Grimm im texte des RF. hat, verteidigen liesse. Die gebräuchliclikeit der letzteren phrase hat Grimm in der anmerk. zu Sendschr. mit einem citate aus Tristan nachgewiesen. 913 das von Grimm eingefuhrte, in PKS. fehlende $\hat{e}$ ist zu streichen. - 914 scheint es mir nicht nötig, mit Schönbach für hât und hâr aus $\mathrm{S}$. houbethâr zu setzen. - 916 sach hätte besser für gesach (PK.) einzutreten. So ist es auch in S. $n u$ statt im mit Schönbach. - 925 hie mit PKS. - 929 tuo, wie S. bietet, und nicht $d \hat{u}$, ef. Schönbach. - 931 f. trotz PKS. (witzen : sitzen) mit Grimm: witze : sitzen. - 938 mit S. wider ôstert, nicht wider hôster (PK.). Für jenes tritt auch Grimm anmerk. im Sendschr. 938 gegen seine fruhere, auf PK. basierte ansicht ein, indem er auf den Roman de Renart verweist. Dem wider ôstert analog ist gên ôstert in den Alddeutschen blättein 1, 249. Nimmit man an der form ôstert anstoss, so kann man etwa ôster setzen. Schönbach greift auf hòster zurïck. - 942 undern statt andern nach S. Vgl. Grimm Sendschr. 8. 58. - 943 der den mit PKS. - 952 var bei Grinm steht nicht in PK. Ich setze mit S. du verst. - 956 ein schlechter vers. Grimms änderung kann ich nicht billigen. Das von ihm für Îsengrîne gebrachte im ist an dieser stelle unklar. Ausserdem sieht man ja, dass der bearbeiter dem dichter, bis auf das reimwort treu geblieben ist. Hier aber tritt 
seine unfahigkeit wider einmal zu tage. Fur sôt musste 955 burne eintreten. Darauf sollte der bearbeiter einen reim finden. Er fand hier, wie später 975 f. nicht nur keinen reinen reim, sondern verlängerte den ursprunglich ziemlich correcten vers (956) auch noch ganz ungebuhrlich. Der vers muss, so schlecht und so lang er ist, stehen bleiben. - 960 in S. besser: dô kam ein bruodir gigân. - $969 \mathrm{f}$. geringe : bertinge. Dieser reim gehört, wie die vergleichung mit $\mathrm{S}$. beweist, dem bearbeiter. Das wort geringe ist fur die heimatsbestimmung des bearbeiters, freilich für keine genaue, von belang. Denn geringe ist, wie ich in meiner abhandlung 'Zur krone Heinrichs von dem Türlîn. Graz 1879 8. 6' nachgewiesen habe, sowol mitteldeutsch, als alemannisch. - $975 \mathrm{f}$. der grund, weshalb der bearbeiter die beiden, in S. guten verse ändert, liegt, wie Schönbach unter hinweis auf 826, richtig bemerkt, in nôtlich. Aber der bearbeiter hat v. 975 des ursprtanglichen gedichtes misverstanden und ein monstrum geschaffen. gotes rache und huoben sich wird man nicht aufgeben durfen, da diese bestandteile auch durch S. gestlutzt werden, über den burnen wider ist des reimes wegen nötig. Grimms fassung ist willkülich und kann aus den entwickelten gründen nicht bestehen. Auch was den inhalt anlangt, hat $\mathrm{S}$. das richtige. Auf die nachricht, dass der wolf in dem brunnen sei, erheben sich die mönche und bereiten sich zum kampfe vor. Nachdem sie hinausgekommen, heben sie sich uber den brunnen. Nach der darstellung des bearbeiters schauen sie sofort nach dem empfange der nachricht in den brunnen und dann erst rlisten sie sich aus. - 976 mit PK. und Schönbach ist der name einzusetzen. wart ein fehler fur was. - 992 was heisst die velt stent? Die erklärung Grinms befriedigt mich nicht. Dagegen stimme ich seiner vermutung R. F. anmerk. bei, wonach es fur diu velt heissen soll diu werlt, aber weiter bin ich nicht für stuont, wie er will, denn das würde dem präs. der cod. und dem folgenden noch nicht entsprechen, sondern stât, wobei man im geiste ergănzen kann, 'wie fruher', um den tubergang $\mathrm{zu}$ den folgenden praeteritis herzustellen. Ich schreibe stât und nicht nach den cod. stêt, da die form mit $\hat{e}$ nicht ein einzigesmal, dagegen die formen stan, $g a n$, öfter durch den reim gestutzt werden. Zu stân vgl. 1084. 1109. 1201. 1379. 1527. 1996. Zu gân 1043. 1090. 1235. 1485. 1512. 
1694. 1742. 1905. 1914. 2088. - $994 \mathrm{f}$. ich halte mich gegen Grimm an die cod. - 999 gra (PK.) uberflüssig. - 1001 mit Schönbach er waenet statt Grimms er sprichet. - 1015 f. zewâre : riundre (PK. reunere). Dieser reim mit nicht umgelauteter $a$ ist wol ebenso von dem bearbeiter aus dem alten gedichte ubernommen worden, wie $1855 \mathrm{f}$. zewâre : verrâtâre (SP. verratere). Ob man freilich darnach fur die sprache des bearbeiters widerstand gegen den umlaut annehmen darf, lasse ich dahingestellt sein. - 1028 streiche ich mit Schönbach als. - 1040 Schönbach nimmt habe aus den cod. auf gegen Grimms hân. Ich lasse in meinem texte das letztere stehen, obwol es hier ziemlich gleichgultig sein mag, ob habe oder hân gesetzt wird. An andern stellen dürfte die volle form doch wol erst den cod. angehören und unnötig den vers verschlechtern. Aus den reimen entnehme ich eine gewisse vorliebe des bearbeiters fur contractionen. Vgl. 175. 361. 369. 396. 439. 460. 515. 575. 672. 723. 807. 1010. 1125. 1277. 1284. 1373. 1404. 1508 . 1510. 1703. 1728. 1840. 1852. 1862. 1940. 1951. - 1042 lat mit Grimm gegen PK. lazet, aus den zu v. 1040 und fruher zu v. 143 gegebenen grinden. Hier ubrigens auch metrisch besser. 1049 schreibt Schönbach mac, doch auch das in PK. uberlieferto moht lässt sich, als erzählend, vertreten. - $1065 \mathrm{f}$. vgl. hierzu oben s. 331. - 1080 iuch von Grimm beigesetzt, ich halte es für uberflussig. - 1114 komma nach quam, mit Schönbach. $1116 \mathrm{zu}$ wan vgl. W. Grimm, Graf Rudolf 8. 19. - 1125 Schönbach setzt (wie 1531) für entschuldeget, entredet hân. Doch bin ich fur enschuldet, das mir formell und sachlich zu passen scheint. entschuldegen kann wie ähnliche bildungen erst durch die schreiber hineingekommen sein. Vgl. die belege bei Lexer. - 1128 liest Paul mit recht fü das mundartlich gefürbte noch der cud. nâch. - $1130 \mathrm{zu}$ Reitze Schönbach. - 1131 Grimm setzt geloube mir, aber warum nicht vernim mir, wie P. bietet? Vernemen wird auch c. dat. (vgl. Lexer) verbunden, wonn freilich gerade im Reinhart sonst die construction mit dem acc. (1387. 1442) vorkommt. K. hat auch an unserer stelle vernim mich (: dir). Ich bleibe bei P. - 1142 ergänze ich nach v. 83 mir. - 1146 mit PK. sich sprechen. Man sieht nicht ein, weshalb Grimm dieses wort durch sich sprâchen ersetzt. In 402 ist sich sprechen sogar im reime (: zebrechen) gebraucht. - 
1157 alles Schönbach. - 1162 vgl. Schönbach. Vorläufig lasse ich die worte stehen, wie sie sind. - 1164 burc mit PK. vgl. oben zu 447. - 1167 der name Reinhart (PK.) ist hier des verständnisses halber nicht unbedingt nötig. Ich bleibe bei dem von Grimm angenommenen er. - 1171 har fur her (im reime auf war), eine alemannische erscheinung, die möglicherweise der bearbeiter auch nur, weil er den reim nicht ändern konnte, beibehielt. Weinhold, Mhd. gr. ${ }^{2} \S 44$ und Al. gr. $\$ 11$ u. 112; Germ. XXIX, s. 274. - $1188 \mathrm{ff}$. ich lasse unentschieden, ob hier der bearbeiter schlecht gestaltet hat oder die uberlieferung schlecht ist und schliesse mich ihr an. Was ubrigens Grimms drabten statt des handschrifthichen quamen anlangt, so ist das hier nicht notwendig, widerholt sich doch 1331.f. quam sehr häufig. Aehnlich ist es $1099 \mathrm{ff}$. - $1189 \mathrm{f}$. vreisam : sân (Weinhold, Mhd. gr. ${ }^{2}$ $\S 216$ und 218). Vgl. ubbrigens vreisam : quam $1273 \mathrm{f} .1835 \mathrm{f}$. $1207 \mathrm{f}$. eine schwierige stelle. Der reim bate : mê ist nicht gut möglich. Wir haben es hier wol mit einem corrumpierten texte zu tun. Die conjectur, die Grimm in der anmerkung autstellt, scheint mir zu gewagt. Wenn ich eine vermutung versuchen dürfte, so wäre es: entriuwen dô der pate schrê, ich enmac gesin niht süener mé. Der reim schrê : mê findet sich im Reinhart öfter. - 1241 warum sollte denn das von PK. uberlieferte der ausfallen? - 1251 schreibe ich mit Lexer (I, 50) âmeizhûfen. - 1272 der hêrre, wie die cod. haben, genligt. Grimm setzt ir hêrre. Ebenso mit PK. burc, wie oben 1164. 1290 bekorn, wie Schönbach. - 1331 mit PK. dar. Diese form auch im reime 1137 und 2139. - 1340 schere, so und nicht stere ist es, wie schon Grimm richtig erkannt hat. Denn 'maulwurf' passt besser zu der im selben verse genannten maus als widder. Zudem wird ja der widder später (1343) erwähnt. - $1401 \mathrm{ff}$. über die confusion vol. Sichönbach. Nach 1427 mit Schönbach doppelpunkt. - 1481 gesach nit den cod. gegen Grimms sach (ohne angabe der varianten). $1489 \mathrm{f}$. wenn Grimm die verse 'unerträglich' fiudet, so soll dem nicht widersprochen werden, aber dem bearbeiter gehören sie trotzdem, man könnte sagen, ebendeshalb an. Vgl. oben zu v. 147. Fir Grimms conjectur in der anmerkung zu RF. 1489, zumal fur entswap, spricht kein ausreichender grund. 1503 algelîche. - 1540 mit PKS. Brûn. - 1541 schreibe ich 
wîstin, wie es auch in S. steht. - 1548 binen. Grimm nimmt aus $P$. 1548 die schwache, 1549 die starke form. Ich wähle consequent die schwache form, welche $\mathrm{S}$. und $\mathrm{K}$. durchaus haben. Die schreibung in P. 1549 biñe würde übrigens eine verschreibung wahrscheinlich machen; vgl. auch Schönbach. - 1568 scheint mir die fassung in $\mathrm{K}$. besser. Doch kann $m e$ in $\mathrm{P}$. bloss verschrieben sein. - 1586 vgl. Schönbach. - 1589 f. S. bestätigt die lesarten von PK. Vgl. Grimm zu Sendschr. 1589. - $1605 \mathrm{f}$. fehlen in S., doch möchte ich nicht sagen, dass sie das alte gedicht schon besessen habe, vgl. Schönbach. - $1623 \mathrm{ff}$. verändert der bearbeiter mehrfach den überkommenen text. 1632 gevolgen nach PK., ebendasselbe wort auch 1634 und 1636. - 1633 das in S. stehende der hirz konnte, wie ich meine, der bearbeiter des bessern verständnisses halber nicht fallen lassen. - 1651 Grimm vermutet in der anmerkung zu RF., dass der dichter sicher künnelinc gehabt habe und das wird nun durch $\mathrm{S}$. bestätigt. Ich behalte für die bearbeitung die ktirzere form bei, obwol sie mittelhochdeutsch blos in den cod. PK. des Reinhart belegt ist. - 1666 ich nehme gegen Grimm die wortstellung der drei handschriften. - 1677 Brân mit PKS. - 1691 ff. vgl. Schönbach. - 1695 nach dem Mhd. wörtb. I, 1024 und Schönbach bloss loch. - 1705 f. unreiner reim êwarte : drate. - 1719 dem ôren, wie in P. und S. - 1722 wie richtig hier die conjectur Grimms ist, geht aus S. hervor. - 1739 boteschaft, wie in S. 1756 gevolgich, wie oben, sowie in S. - 1757 al umbe mit Schönbach. - 1758 nach Grimm und Schönbach zog der bearbeiter die unreinheit des reimes (uberschussiges $-n$ ) dem fehlen des artikels vor. Auch ich mag den uberlieferten text nicht ändern. Doch kann ich den gedanken nicht unterdrucken, dass der bearbeiter bei seinem offenbaren streben nach reinen reimen und seiner accomodation an die sprache des dichters immerhin einen vers angenommen haben könnte, der ihm nicht nur einen reinen reim bot, sondern auch einen sprachlichen ausdruck, der dem dichter correct war. - $1770 \mathrm{f}$. PK. Laben hier offenbar corrumpiert, wie schon das unpassende geswachen beweist. - 1771 oder mit S. - In 1772 haben PK., abgesehen vom versschluss, die wortfolge von S. In dieser handschrift ist ubrigens der text so gut, dass ich denselben unbedenklich eingesetzt habe. P. hat nach meiner collation nicht machen, 
wie bei Grimm steht, sondern auch lachen wie $\mathrm{K}$. uberliefert. - 1773 auch an dieser stelle scheint der text in PK. verderbt. Ich greife deshalb auch hier auf den bessern text in S. zurtkck. - 1776 nehme ich mit Schönbach die ganze uberlieferung aus PK. auf, obwol das einen ungebuhrlich langen vers gibt. 1779 dîn neve mit PKS. gegen Grimm: der neve dîn. - 1781 vgl. Schönbach. - 1786 P. schreibt de, K. die fur der Gl. Das eine oder das andere könnte dem bearbeiter angehören, dem dichter kaum. Vgl. Weinhold, Mhd. gr. ${ }^{2}$ \$ 482. Doch bieten 2250 P. und K. der Gl. - 1787 mit Grimms conjectur iu künde gît, gewaerlich kann ich mich nicht einverstanden erklären. gewaerlich wird durch alle drei cod. uberliefert und durch den reim gestutzt. Aber von dem durch PK. gebotenen zweiten satz wen si sint gewerlich durfte doch mehr aufzunehmen sein, als das letzte wort. In der auch an dieser stelle lefecten handschrift $S$. ist uns von dem ganzen verse blos gewarlich erhalten. Jedoch die räumliche ausdehnung der verse ins auge gefasst, kann der vers mehr worte und buchstaben enthalten haben, als iu kunde gît gewârlich. Grimm hat das auch gefublt, in dem or im Sendschr. diesen vers so restituiert: iu kunde gît vil gewärlich. Ich habe aus dem text von PK. si sint gewaerlich aufyenommen. Einen ganzen satz statt gewaerlich anzusetzen, wird sich wol auch deshalb empfehlen, weil der nach. druck auf die wahrheit der mitteilung gelegt werden soll. Mit Grimm schreibe ich $g \hat{\imath} t$, da ich doch nicht wage, das von PK. uberlieferte mundartliche geit (Weinhold, Mhd. gr. ${ }^{2}$ § 53), das auch blos den cod. angehören könnte, in den text zu setzen. ${ }^{1}$ ) - 1796 krimel mit PKS. Naturlich mit Grimm vuor, wio S. gegen ver in PK. beweist. - 1818 drinne wie PK. 750. - 1826 arzat auf grund der reime 1915 f., 1923 f., 1962 f. -1837 ausser dem reime spricht auch $S$. für albesunder. -- 1844 nach PKS. solde (Grimm tohte). - 1852 acceptiere ich Grimms wortstellung lasters vil. - 1858 hatte S. wol den ganzen langen vers 80, wie ihn PK. bieten. Einige worte sind uns aus S. erlialten (er sprach - wizzin wol dáz ir), andere (kunec wir sint) lassen sich erschliessen. Ich behalte den vers bei, wie

1) Aehnliche contractionen sind in reimen nicht selten: geseit 72. 318. 323. 536. 598; unverseit 717 ; geleit 363 ; treit 1824; lât 308 . 
er in PK. steht und lese ihn mit zweisilbiger senkung. Oder sollen wir etwa er sprach weglassen? $\mathrm{Zu}$ sint, 2. pers. plur. praes., al.-md. form, vgl. Weinhold, Mhd. gr. ${ }^{2}$ § 364. - 1863 rabe. Den ausgang auf $e$ bieten P.S. gegen Grimm RF. raben. Fur rape, wie S. hat oder rappe, wie Grimm Send. setzt, kann ich mich nicht entscheiden, so bleibe ich bei PK. - 1864 henket mit rücksicht auf $1853 \mathrm{f}$. - 1872 setze ich er mit S. gegen man in PK. Weshalb sollte denn auch der bearbeiter das bessere er gegen man vertauscht haben? - 1874 Bendin. S. hat an der einzigen stelle, an der daselbst das wort erhalten ist (1886), bendin und PK. Laben an derselben stelle die gleiche schreibung. - 1884 al wie 1731. - 1891 liez mit PK. oder lie mit Grimm? Fur das eine, wie für das andere finden sich reime und so bleibe ich, da die reimbeweise fur das erstere ( $35 \mathrm{f}$., $783 \mathrm{f}$., $1549 \mathrm{f}$ ) nicht zwingender sind, als fur das letztere (41 f., 811 f., 935 f., 991 f., 2075 f.), bei Grimms lie. - 1908 setze ich mit P. das in K. fehlende wider iuch, da ein ähnlicher ausdruck 1955 an der stelle des reimes steht. Man beachte hier den reim leben : geben und $2007 \mathrm{f}$. sagen : getragen, also die 1. pers. sg. praes. mit $-n$ gebildet. Der bearbeiter kann diese form aus dem alten gedichte ubernommen oder selbst gebildet haben. Dass K. die varianten leben | sol und sage und P. lebe bietet, whrde kein grund dagegen sein. Im alemannischen findet sich der ausgang auf -n seit dem 12. jahrhundert. Vgl. Weinhold, Mbd. gr. ${ }^{2} \S 367$, Al. gr. 339. Im frănkischen ist diese form auch schon frubo nachweislich. Cf. Weinhold, Mhd. gr. a. a. o. - 1910 Grimms wortstellung, meister aus PK. - $1919 \mathrm{zu}$ dem handschriftlichen iz im vgl. Schöbach. - 1929 f. entwichen : begrifen, unreiner reim. Oder durften wir ihn als mundartlich rein auffassen? Vgl. Weinhold, Mhd. gr. ${ }^{2} \S 233 \mathrm{ff}$. - 1964 azer maze, das PK. uberliefert, statt des von Grimm angesetzten unmazen. - 1968 müeste auf Pauls vorschlag. - 1972 lies mit Schönbach armman. - 1974 nehme ich die contrahierte form hân wir. 1980 ich mit Schönbach als versehen zu streichen. - 2009 fur gewermet der cod. gewarmt mit rucksicht auf den durch reime sonst nachgewiesenen ruckumlaut - $2014 \mathrm{im}$, das ich mit PK. ansetze, ist hier, wie $2088 \mathrm{er}$, der vorlăufer des substantive. Ich schreibe katzenhat, da ich die zusammensetzungen mit katze bei 
Lexer so finde. - 2022 bleibe ich der uberlieferten wortstellung etwas mehr treu, als Grimm. - 2041 krouch mit Paul. - 2054 mit Schönbach er tet. - 2099 schreibe ich, wie 1333 - PK. hat hier, elephant - elefant. Ebenso auch 1103 und 1635. - 2123 zem Erstein mit Grimm, Altd. bl. I, 417. - 2140 ir mit Schönbach. - 2143 schreibe ich künden für kündigen, da letzteres erst der späteren zeit, hier dem schreiber, anzugehören scheint. Lexer I, 1773 und D. Wtb. V,2629f. Vgl. oben zu v. 1125. - 2146 und 2150 nehme ich ebtissin, wie in $P$. an beiden und in $K$. an letzterer stelle steht. Dieselbe form etwa auch 2123 ? Vgl. Schönbach. 2149 was soll schriten bei Grimm heissen? Noch dazu ein praesens umgeben von praeteritis! $\mathrm{PK}$. überliefern schreiten. Das ist mundartlich soviel als schrieten, praet. von schrôten, hauen, schneiden. Das wort wurde auch zu dem folgenden gut passen. ei für gemeindeutsches ie ist nach Weinhold, Mhd. gr. ${ }^{2} \S 131$ und 136 besonders im westen des mitteldeutschen gebietes verbreitet. Vgl. noch Germ. XXIX s. 268. Dem bearbeiter kann schreiten angehören, dem dichter aber nicht da sich im elsässischen ei für ie nach Weinholds, Al. gr. § 131 nur vom 14-16. jahrhundert und auch in dieser zeit nur zuweilen findet. Uebrigens könnte schreiten auch $=$ schrieten, 3. pers. plur. prät. von schrien. Weinhold, Mhd. gr. ${ }^{2} \S 425$. - 2217 f. 'flickreime' nach Grimm, die er beseitigen möchte. Dieselben mlissen aber nicht einmal erst von dem bearbeiter, sondern können bereits von dem dichter herruhren. Denn den reim $d \hat{a}: s \hat{a}$ gebraucht auch der dichter. Vgl. s. 917 f., 963 f., 1589 f. Aus der bearbeitung vgl. ubrigens noch $1729 \mathrm{f}$. (S. $d \hat{d}: g \hat{a})$. -2230 das md. hin weck in PK. gehört wol erst den handschriften an, weshalb ich mit Grimm dafur enwec setze. - 2248 Schönbach vermutet dem rôten $\mathrm{R}$. - Nach 2248 bringen PK. zwei verse, die Grimm nicht in den text aufnimmt. Ich möchte dieselben nicht fallen lassen, denn sie können gar wol den schluss des alten gedichtes gebildet haben. Ueberdies berühren sie sich nicht blos in ihrem tone, sondern zum teil auch inhaltlich mit versen, die Grimm anstandslos in den text gesetzt hat und die sich auch in S. finden. Man vgl. $854 \mathrm{f}$., $1791 \mathrm{f}$., auch 1787. 2255 halte ich ouch dem sinne nach für uberflussig.

GRAZ.

KARL REISSENBERGER. 